\title{
Erythema nodosum leprosum and active leprosy after ChAdOx1-S/nCoV-19 recombinant vaccine. A report of two cases
}

\author{
Paula Frassinetti Bessa Rebello ${ }^{\text {a }}$ \& Silmara Navarro Pennini ${ }^{\text {a }}$ \\ ${ }^{a}$ Tropical Dermatology Outpatient Clinic - Fundação de Dermatologia \\ Tropical e Venereologia Alfredo da Matta, Manaus, State of Amazonas, \\ Brazil \\ ORCIDs: https://orcid.org/0000-0002-9503-7185; https://orcid.org/0000-0 \\ 001-8102-3885
}

Submitted 25 July 2021; Accepted 30 September 2021

\begin{abstract}
Summary Leprosy reactions are acute inflammatory processes, immunologically mediated that may be precipitated by many factors, including infections and vaccines. They are classified in Type 1 Reaction or Reversal Reaction, and Type 2 Reaction (T2LR). The most common clinical manifestation of T2LR is the erythema nodosum (EN), and, because of this, it is also called Erythema Nodosum Leprosum (ENL). Reactions can occur before, during or after the diagnosis and treatment of leprosy. We present two cases of ENL in patients who were released from treatment, and had received the first dose of ChAdOx1-S/nCoV-19 vaccine just a few days before the appearance of the skin lesions. One of them also had signs of active multibacillary leprosy. Reports of erythema nodosum related to COVID-19 disease have been published, but to our knowledge, this report is the first to describe cases of ENL related to SARS-CoV-2 vaccines.
\end{abstract}

Keywords: COVID-19, SARS-CoV-2, ChAdOx1nCoV-19, multibacillary leprosy, erythema nodosum leprosum, type 2 leprosy reaction

\section{Introduction}

Type 2 Leprosy Reaction (T2LR) includes erythema nodosum (EN) as the most common clinical manifestation, with painful erythematous nodules as well as systemic manifestations like fever and malaise, sometimes accompanied by peripheral neuritis, oedema, iritis or orchitis. Because of these specific features, T2LR is also called Erythema Nodosum Leprosum (ENL). It is considered an immune complex syndrome with increased levels of TNF- $\alpha$ and IFN- $\gamma$. It occurs in multibacillary patients, mainly those classified as lepromatous leprosy (LL), during its natural course, during treatment or after it. ${ }^{1-3}$

The precipitating factors for ENL reactions are: LL classification (Ridley-Jopling), bacillary index (BI) higher than $4+$ and, among others, infections and vaccines. ${ }^{4-6}$

Correspondence to: Paula Frassinetti Bessa (e-mail: paularebello64@gmail.com) 


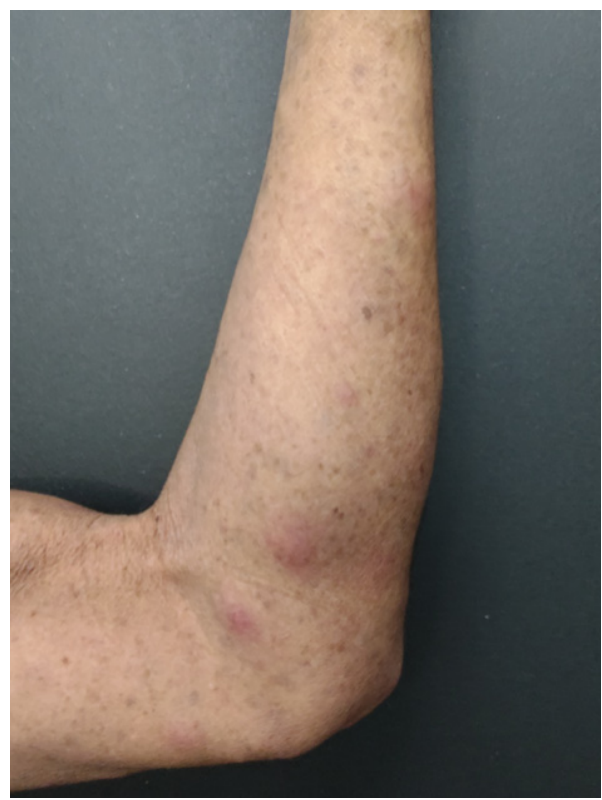

Figure 1. Case 1-Erythematous nodules on the upper limb.

During a pandemic, it is expected that both the disease and its vaccines can be associated with immunological reactions. Reports of erythema nodosum related to COVID-19 disease have been published, but to our knowledge, this is the first report to describe cases of ENL related to SARS-CoV-2 vaccines. ${ }^{7-9}$ The cases occurred in a referral centre in Manaus, State of Amazonas, Brazil, an endemic area for leprosy.

\section{Case reports}

CASE 1

A 44-year-old male who had been treated for LL leprosy (BI of 5+ with solid bacilli) with 12-dose multibacillary multidrug therapy (MDT), as recommended by the World Health Organization (WHO). ${ }^{10} \mathrm{He}$ was released from treatment without any skin lesions characteristic of leprosy and with a BI of 3.5+ (fragmented and granular bacilli). In the three months after completing this treatment he returned to the service with disseminated erythematous nodules (Figure 1) accompanied by fever. He had received the first dose of the ChAdOx1-S/nCoV-19 vaccine five days earlier and the lesions started on the third day after the vaccination. He had never presented with any leprosy reaction before. He was then diagnosed with ENL and started on thalidomide $100 \mathrm{mg} /$ day, which is the drug of choice for the treatment of males, in Brazil. After fifteen days he returned without skin lesions.

\section{CASE 2}

A 43-year-old male, had received 24-doses of MDT/WHO for Borderline Lepromatous Leprosy (BL), 25 years ago. He reported that he was without any skin lesions until two days after receiving the first dose of the $\mathrm{ChAdOx} 1-\mathrm{S} / \mathrm{nCoV}-19$ vaccine. He stated that from that point onwards he had developed a fever, malaise, and painful nodules. On clinical examination, 


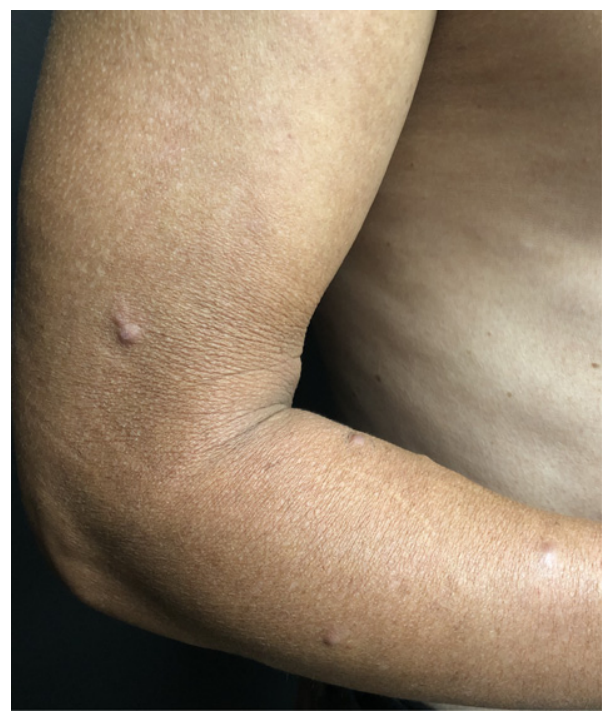

Figure 2. Case 2-Skin-coloured cutaneous papules (lepromas).

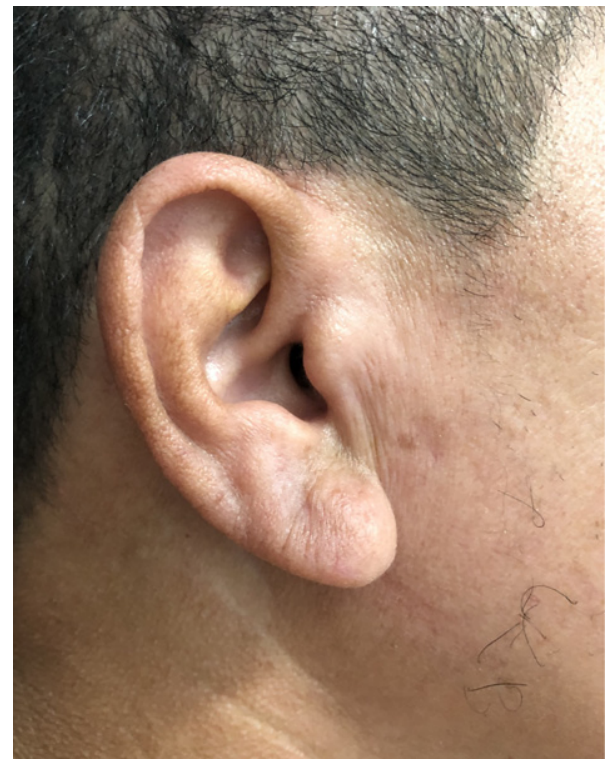

Figure 3. Case 2-Earlobe infiltration.

there were painful erythematous nodules disseminated on his body, compatible with ENL, some dome-shaped, skin-coloured to coppery-red papules (lepromas) and mild infiltration of the ear and face (Figures 2 and 3). Skin smear microscopy was positive with the presence of solid bacilli and a BI of 3.75. A skin biopsy was performed to confirm the diagnosis of leprosy by histopathology and to investigate drug resistance by Polymerase Chain Reaction (PCR). The histopathological examination demonstrated the epidermis with acanthosis and 


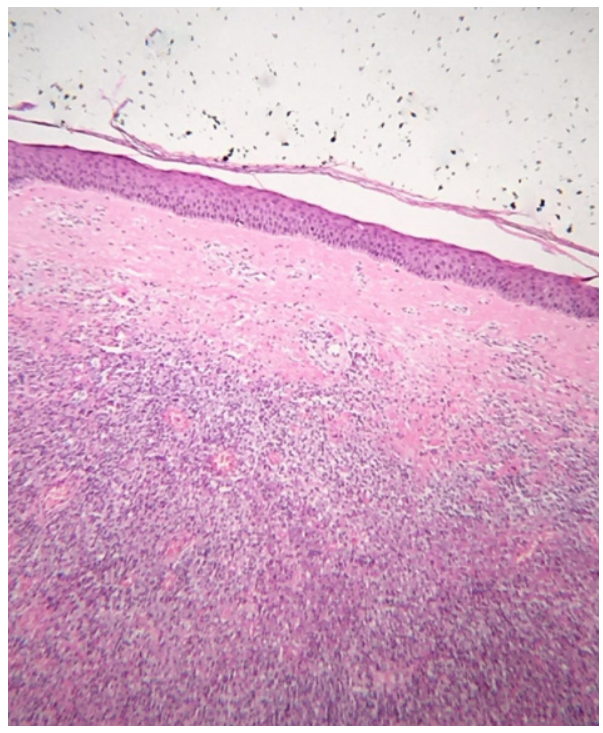

Figure 4. H\&E-stained skin biopsies of case 2-reticular dermis with diffuse inflammatory infiltrate involving vessels and adnexa.

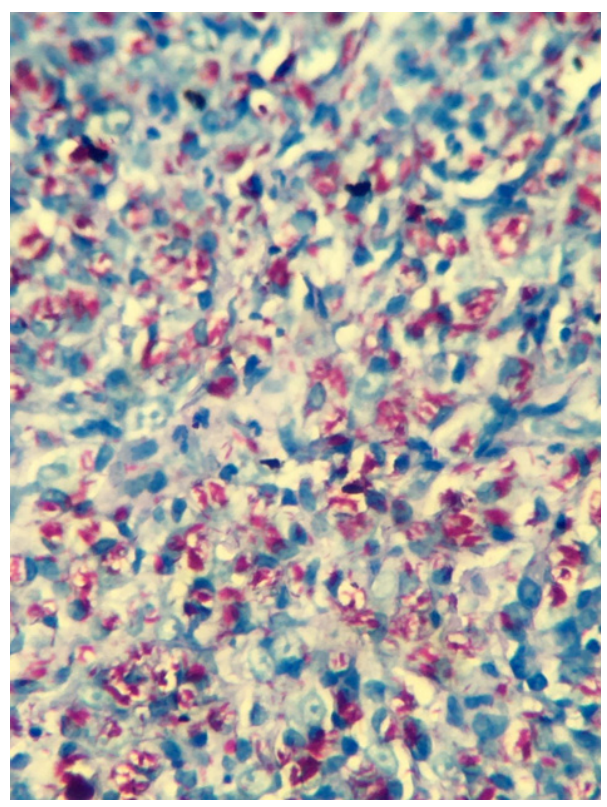

Figure 5. Solid bacilli grouped in globi (Wade's stain).

hyperkeratosis, the reticular dermis with diffuse inflammatory infiltrate involving vessels and adnexa, composed of lymphocytes, neutrophils and vacuolated histiocytes containing solid bacilli grouped in globi. The bacilli were also found inside nerve filaments, sweat glands, endothelium, vascular wall and lumen, and in adipocytes (Figures 4 and 5). He was 
treated with $100 \mathrm{mg} /$ day of thalidomide and started MDT while waiting for the result of the investigation into possible drug resistance.

\section{Discussion}

We describe two cases of ENL that appeared after receiving the first dose of ChAdOx1$\mathrm{S} / \mathrm{nCoV}-19$ vaccine and in one of them it was possible to diagnose active leprosy.

It is not rare to diagnose cases of leprosy when patients have a leprosy reaction. The acute inflammatory signs and symptoms of the reactions motivate those who have not noticed the insidious and chronic leprosy skin lesions to look for health assistance.

In general, the diagnosis of ENL is based on classic clinical symptoms. ${ }^{11}$ However, EN may be a clinical manifestation of other diseases or an adverse effect of drugs or vaccines. The clinical difference between them is the body distribution of the lesions. In ENL, the nodules occur in any region, while the EN due to other causes has predilection for the shins. ${ }^{3}$

The ChAdOx1-S/nCoV-19 vaccine is a monovalent vaccine, composed of a single vector recombinant chimpanzee adenovirus, deficient for replication (ChAdOx1), which expresses the glycoprotein $\mathrm{S}$ of SARSCoV-2. After administration, SARS-CoV-2 glycoprotein $\mathrm{S}$ is expressed locally by stimulating the cellular immune system and neutralizing antibodies, with high levels of TNF- $\alpha$ and IFN- $\gamma \cdot{ }^{12}$

The immunologic mechanisms involved in the association between vaccines and leprosy reactions have not been fully described; however, TNF- $\alpha$ and IFN- $\gamma$ are also the main mediators present in ENL. ${ }^{3,10}$

The most common systemic adverse reactions of the ChAdOx1-S/nCoV-19 vaccine are: fatigue (70\%); headaches (68\%); malaise (61\%); muscle ache $(60 \%)$; chills $(56 \%)$; and feeling feverish $(51 \%) .^{12}$

Both these more frequent and those rarer side effects, such as ENL described here, do not invalidate the vaccine's safety or its efficacy. The benefits of the vaccines, individually and in terms of public health, have been proven with the reduction of symptomatic COVID-19, and with further protection against severe disease. ${ }^{13}$

\section{Conclusion}

Mass immunization for the prevention of COVID-19 is necessary and has been carried out worldwide. However, health professionals, mainly in leprosy endemic countries, need to be made aware of the occurrence of erythema nodosum leprosum that may appear in patients that are vaccinated. They have to look for the signals of active leprosy in these patients for a possible diagnose of a new case of leprosy, relapse or reinfection.

\section{Ethics}

This case report was approved by the FUAM Research Ethics Committee (CAAE 50023521.1.0000.0002).

\section{Acknowledgements}

We acknowledge Professor Simon Jacobs for the language proof reading and Dr. Patricia Morais, dermato-pathologist, for the histopathological description and photos. 


\section{References}

1 Ramos-e-Silva M, Rebello PFB. Leprosy recognition and treatment. Am J Clin Dermatol, 2001; 2(4): $203-211$.

2 Penna GO, Gonçalves HS, Lastoria JC, Machado PRL, Talhari S. Reações Hansênica. In: Talhari S, Neves RG, Penna GO, de Oliveira MLV (eds), Hanseníase. 5th edn, Manaus: Editora DiLivros, 2015; pp. 45-60.

3 Naafs B. Leprosy reactions. Trop Geogr Med, 1994; 46(2): 80-84.

4 Scollard DM, Smith T, Bhoopat L et al. Epidemiologic characteristics of leprosy reactions. Int J Lepr Other Mycobact Dis, 1994; 62(4): 559-567.

5 Manandhar R, LeMaster JW, Roche PW. Risk factors for erythema nodosum leprosum. Int J Lepr Other Mycobact Dis, 1999; 67(3): 270-278.

6 Cohen PR. Combined reduced-antigen content tetanus, diphtheria, and acellular pertussis (TDAP) vaccinerelated erythema nodosum: case report and review of vaccine-associated erythema nodosum. Dermatol Ther (Heidelb), 2013; 3: 191-197.

7 Suter P, Mooser B, Pham Huu Thien HP. Erythema nodosum as a cutaneous manifestation of COVID-19 infection. BMJ Case Rep, 2020; 13(7): e236613.

8 Ordieres-Ortega L, Toledo-Samaniego N, Parra-Virto A et al. Atypical erythema nodosum in a patient with COVID-19 pneumonia. Dermatol Ther, 2020; 33(4): e13658.

9 Sipfle DON, Bridwell MD RE, Roper DOJ. Erythema nodosum-like rash in a COVID-19 patient: A case report. Am J Emerg Med, 2021; 40: 227: e1-227.e2.

10 WHO Study Group on Chemotherapy. Chemotherapy of Leprosy: Report of a Study Group. WHO Technical Report Series, 847, 1994.

11 Negera E, Walker SL, Girma S, Doni SN, Tsegaye D, Lambert SM, Idriss MH, Tsegay Y, Dockrell HM, Aseffa A, Lockwood DN. Clinico-pathological features of erythema nodosum leprosum: A case-control study at ALERT hospital, Ethiopia. PLoS Negl Trop Dis, 2017; 11(10): e0006011.

12 Folegatti PM, Ewer KJ, Aley PK et al. Safety and immunogenicity of the ChAdOx1 nCoV-19 vaccine against SARS-CoV-2: a preliminary report of a phase 1/2, single-blind, randomised controlled trial. Lancet, 2020; 396(10249): 467.

13 Lopez Bernal J, Andrews N, Gower C, Robertson C, Stowe J, Tessier E, Simmons R, Cottrell S, Roberts R, O’Doherty M, Brown K, Cameron C, Stockton D, McMenamin J, Ramsay M. Effectiveness of the PfizerBioNTech and Oxford-AstraZeneca vaccines on covid-19 related symptoms, hospital admissions, and mortality in older adults in England: test negative case-control study. BMJ, 2021; 373: n1088. 